Dong, Z., Abdulghani, A. M., Imran, M. A. and Abbasi, Q. H. (2020) Artificial Intelligence enabled Smart Refrigeration Management System using Internet of Things Framework. In: 2020 International Conference on Computing, Networks and Internet of Things (CNIOT 2020), Sanya, China, 24-26 Apr 2020. ISBN: 9781450377713

There may be differences between this version and the published version. You are advised to consult the publisher's version if you wish to cite from it.

CInternational Conference on Computing, Networks and Internet of Things.This is the author's version of the work. It is posted here for your personal use. Not for redistribution.

http://eprints.gla.ac.uk/214995/

Deposited on: 5 June 2020

Enlighten - Research publications by members of the University of Glasgow http://eprints.gla.ac.uk 


\section{Artificial Intelligence enabled Smart Refrigeration Management System using Internet of Things Framework}

\author{
Zhongxu Dong \\ James Watt School of \\ Engineering \\ University of Glasgow \\ Glasgow, UK \\ 326691586@qq.com
}

\author{
Amir M. Abdulghani \\ Smart City Research Group, \\ Sultan Qaboos University, \\ Oman \\ \& James Watt School of \\ Engineering \\ University of Glasgow \\ Glasgow, UK \\ amirmohamed.abdulghani@g \\ lasgow.ac.uk
}

\author{
Muhammad A. Imran \\ James Watt School of \\ Engineering \\ University of Glasgow \\ Glasgow, UK \\ Muhammad.Imran@glasgow. \\ ac.uk
}

\author{
Qammer H. Abbasi \\ James Watt School of \\ Engineering \\ University of Glasgow \\ Glasgow, UK \\ qammer.abbasi@glasgow.ac. \\ uk
}

\begin{abstract}
Design of an intelligent refrigeration management system using artificial intelligence and Internet of Things (IoT) technology is presented in this paper. This system collects the real-time temperature inside the refrigeration implement, record the information of products and enhance function of refrigerators through the application of Internet of Things technology to facilitate people in managing their refrigerated and frozen groceries smartly. The proposed system is divided into two parts, On-board sub-system and Internet based sub-system. An Arduino Leonardo board is used in onboard sub-system to control other components including low power machine vision OpenMV module, temperature \& Humidity sensor, and GY-302 light intensity sensor. OpenMV camera module is used for recognizing types of food, reading barcodes and OCR (optical character recognition) through convolution neural network (CNN) algorithm and tesseract-ocr. The food type identification model is trained by the deep learning framework Caffe. GY-302 light intensity sensor works as a switch of camera module. DHT11 sensor is used to monitor the environmental information inside the freezer. The internet based sub-system works on the things network. It saves the information and uploads it from onboard sub-system and works as an interface to food suppliers. The system demonstrates that the combination of existing everyday utility systems and latest Artificial Intelligence (AI) and Internet of Things (IoT) technologies could help develop smarter applications and devices.
\end{abstract}

\section{KEYWORDS}

Internet of Things (IoT), Artificial Intelligence (AI), Machine Vision, Convolution Neural Network, The Things Network, Smart City, Smart Home Application

\section{INTRODUCTION}

The recent trend and desire to modernize cities and the way people living in those smart cities perform their tasks, including considerably basic to advanced complex processes, has led to the need to enhance and develop intelligent tools, products and processes. This includes home appliances such as refrigerators and other household equipment. Refrigerator at homes, since its invention, has been used as a refrigeration equipment only. Managing and keeping track of its contents is done manually by the users as the equipment does not have any intelligence or ability to manage automatically on its own. An experiment in 2019 displays that the U.S. households waste a substantial quantity of food because there are no effective ways to grasp all types and states of food saved in domestic refrigeration devices [1]. Therefore, it is necessary to integrate the household refrigerator with Internet of Things framework and Artificial Intelligence technology to try and automate the management of these appliances to hopefully help in reducing food wastage.

The purpose of Internet of Things (IoT) is to open additional channels for us to remotely control the physical world [2]. The purpose of this network is for realizing the ubiquitous connection of objects and objects, objects and people and realize intelligent perception, identification and management of objects and processes through all kinds of possible network access. Specific to this system, the purpose is that treating the things network as an interface to share the information saved in it with the end-user and food suppliers. The user will receive message about the state of their fridge and freezers. Meanwhile the food supplier can also track the logistics more accurately through the shared information. At the stage of information collection, temperature and humidity are regarded as the important indicators of refrigerator's environment. Most domestic refrigerators operate at $7{ }^{\circ} \mathrm{C}$. If products are desired to be kept refrigerated for longer, the temperature should be adjusted to $4{ }^{\circ} \mathrm{C}$ [3]. Senor DHT11 will monitor these parameters and upload to The Things Network. End-users obtains this information in real time and choose the appropriate operating mode. Meanwhile, it is significant to take the information of stored food such as type, barcode and expiration date. This function relies on the convolution neural network algorithm built into the OpenMV camera module. The test model is trained by deep learning framework Caffe. However, recognizing the expiration date printed on the packaging surface has higher requirement on algorithm, picture and processor. Thus, OpenMV will just take the image. Then, we have used the tesseract-OCR application for date recognition. All this information is then uploaded to the things network. 
Barcode of every product is unique which stores product's production information [4]. Through the things network, barcode uploaded from every single domestic refrigerator can contact and communicate with the database of food suppliers. Based on that, various related applications can be developed. For example, the businesses could analyze product sales more accurately as well as customers can realize more basic information about the product such as expiration date and origin.

The main difficultly in achieving the intelligent management of domestic refrigeration devices is that there is no uniform packaging for stored items. Therefore, serval different recognition methods are developed in this system including image identification, barcode reading and optical character recognition. However, with the support of Internet of Things, the end-user could obtain the list of stored items and the expiration date of them through the Ubidots interface. This will help to provide the guidance to users for more rational shopping and to avoid unnecessary waste of food stored in the refrigerators.

\section{LITERATURE REVIEW}

This section highlights the need and sets the stage for doing more research in the field of refrigeration system management, followed by the effect of temperature on food wastage and application of bar codes to help store information about the contents of the refrigerator. The discussion on sensor, techniques used for image and feature recognition, Convolutional Neural Networks (CNN), and Machine Vision (MV) device is included in the later parts of this section to provide the rationale behind the approach, components and techniques adopted in this work.

The information provided in document [1] shows that there is little research on management of household refrigerators, and more research in this domain is needed to help reduce food wastage. The literature investigates the list of household refrigerators used by US consumers to evaluate the connection between daily procedures and processes related to food and food wastage. Experimental results demonstrate that forgetting the expiration date of food is one of the major causes of food wastage. Several studies presented in [3] have also demonstrated that lowering the temperature to $4{ }^{\circ} \mathrm{C}$ can significantly extend food's storage life. According to current British waste statistics, reducing the temperature from $7{ }^{\circ} \mathrm{C}$ to $4{ }^{\circ} \mathrm{C}$ can save $£ 162.9$ million in waste annually [3]. Quantitative data in this [3] also shows a unblemished correlation between refrigerator's temperature and the food's storage life. Hence, highlighting the necessity to monitor the temperature for optimizing the storage life of food.

In reference to the usage of barcodes for storing and retrieving information about the products, literature in [4] shows as to what level the application of barcodes has enhanced the internal logistics issues related to hospital pharmacies and using bar codes to store unique information of numerous products is very practical. For monitoring and controlling the temperature of refrigeration equipment some sensing components are required. A food management system based on smart refrigerators was developed in [5]. The system includes database initialization module, food information input module, storage module, alarm module, food recommendation module, query module and network access module. User must manually enter food data and the system would then recommend the nutritionally balanced menu to the user.

Another refrigerator food management scheme was developed as part of work presented in [6]. In this, food was marked by the wireless communication identification tag card. On-system installed label detection system was used in the refrigerator to read label in order to obtain food-related information. The information on the food label is compared with the information stored on the Internet. It is convenient to realize the control of food types only through networked labels, but the main shortcoming is that one needs to customize labels for each food item.

In [7] DHT11 to detect the temperature of the internal environment of the greenhouse has been used. This article presents detailed analysis of the process of measuring temperature and humidity by digital temperature and humidity sensor DHT11. We have used this sensor in the prototype developed and presented here. The sensor can achieve the response speed of sending temperature and humidity data with accuracy to two decimal places once every minute. The results show that the node composed of DHT11 has small volume, high accuracy and fast response speed.

Accuracy and relevance of convolutional neural networks in image recognition has been verified in [8]. The effects of several different image recognition algorithms on food recognition were compared as well. The advantage of convolutional neural networks is that in the convolutional layer it usually contains multiple feature planes. Each feature plane consists of neurons separated by rectangles. Neurons in the same feature plane share weights. The weight shared here is the convolution kernel. Since the convolution kernel obtains reasonable weights during the network training process, it reduces the connections between the various layers of the network and reduces the risk of overfitting. The experimental results also show that the recognition accuracy of CNN group is high. Convolution and subsampling (merging) greatly simplifies the complexity of the model and reduce the model parameters. This is very crucial and highly desirable for writing image recognition programs that can run on $\mathrm{O}$ penMV smoothly without constraining the memory resources on board. Research presented in [9] indicates to the feat of convolutional neural networks in solving image classification problems. In this research, the convolutional neural network model of the winner of the ilsvrc12 competition was implemented. Around 1.2 million images and 1000 categories are distinguished by this method successfully. The application is executed using the Caffe library. Studies have shown that the Caffe deep learning framework that can call GPU operations and has a Python interface has the characteristics of simple use and high training efficiency. In view of the excellent performance of CNN neural networks in [8] and [9]. Use it to train models and run related programs in the camera module OpenMV to achieve the purpose of quickly and accurately identifying food types and barcodes.

Several algorithms were analyzed for processing RGB images into binary grayscale images in [10] suggesting that grayscale images can enhance the accuracy of image recognition. The maximum value method, the average value method or the weighted average 
value method can convert the three-channel RGB image into a single-channel grayscale image. However, the grayscale image still maintains the gradient information between pixels. This conversion greatly reduces the amount of data in the image and retains the gradient information to improve the efficiency and accuracy of image recognition.

\section{MATERIALS AND METHODS}

The components involved in freezer management system are list as follows.

- An Arduino Leonardo microcontroller

- An OpenMV M4 H7

- A DHT11 Temperature \& Humidity sensor

- A GY-302 light intensity sensor

The management system consists of two parts: On-board subsystem and Internet based sub-system. The On-board sub-system focuses on the information collecting and analysis. The circuit of this part fixes inside the refrigerator. The Internet based subsystem is applied to save data and achieve the function of interacting with end-users and food supplier. The architecture of the system is shown in figure 1 below.

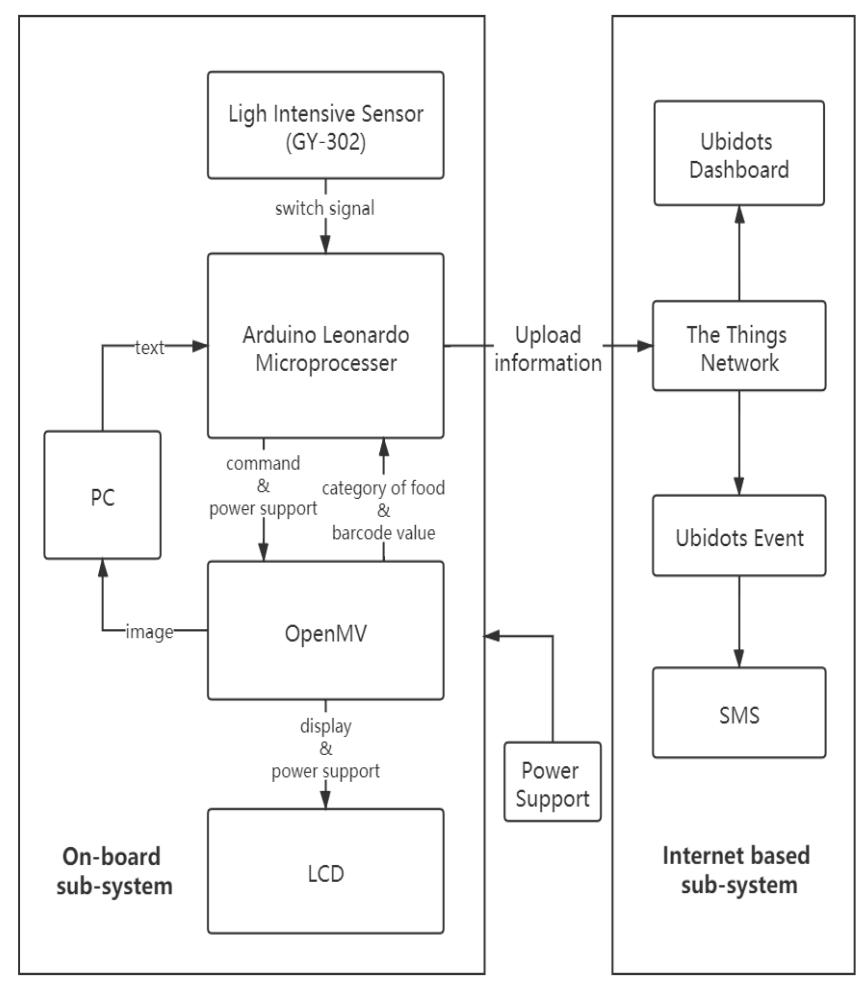

Figure (1) The architecture of the system

The program composes three sections which are microcontroller program, computer program and network program. Program run in microcontroller drives all sensors and modules to collect information and analysis. Then the result is transmitted to and saved at Gateway Console. Temperature and humidity will be shown in Ubidots Dashboard. The event such as the stored chocolate has expired will be noticed by sending message. Tesseract built in computer realizes the function of Optical Character Recognition. In this way, the expiration date can be recorded.

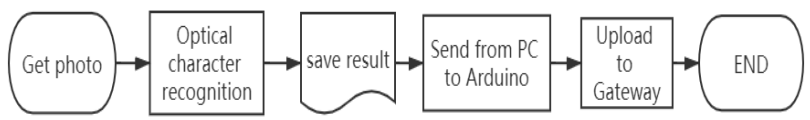

Figure (2) The flowchart of optical character recognition

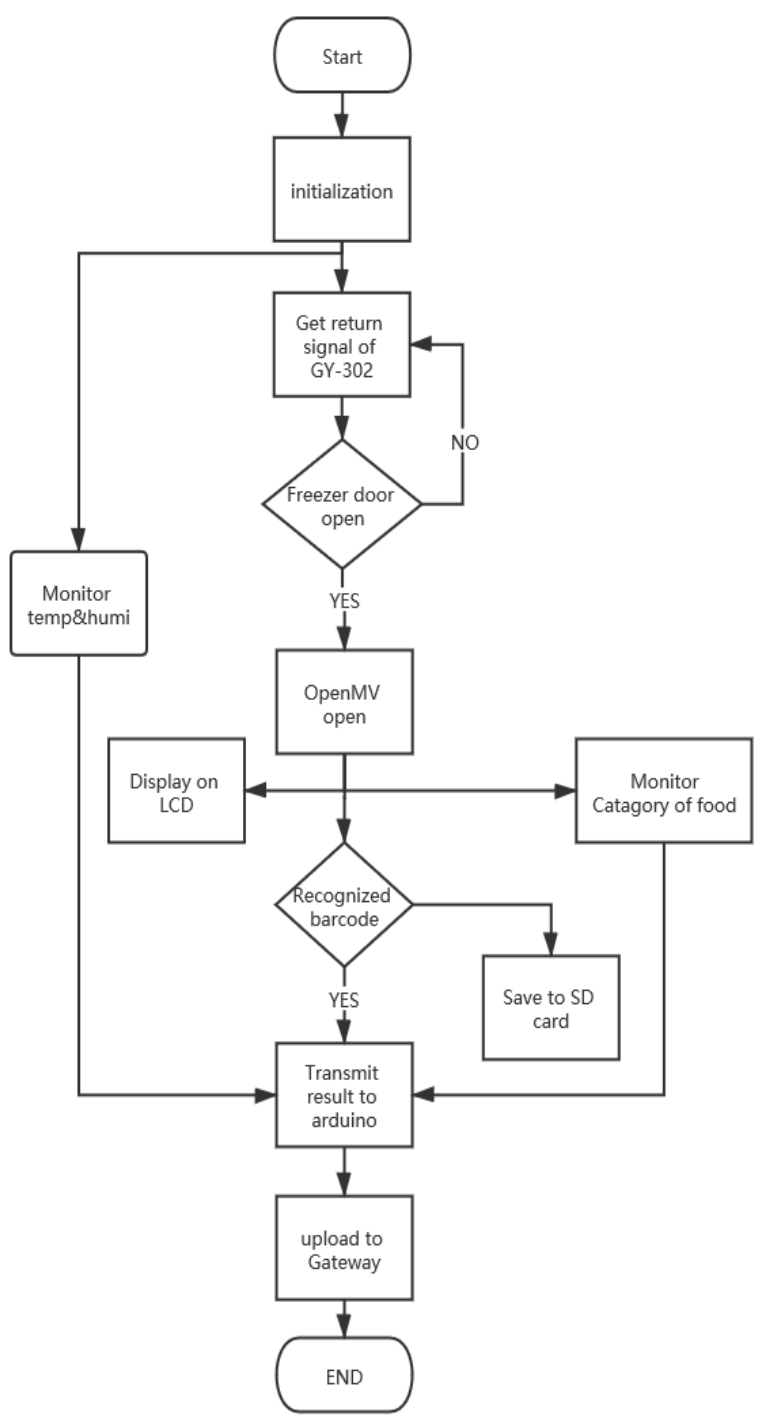

Figure (3) The flowchart of On-board sub-system In this part of the operating logic of the system light intensity sensor works as the switch of OpenMV. So that the camera 
module operates only when the refrigerator door is open. At the same time, DHT11 collects data continuously.

\section{SYSTEM ANALYSIS}

This system developed for refrigerator composed by Arduino Leonardo board, light density sensor, DHT11 sensor, camera mode and the thing network. The hardware circuit diagram is shown below.

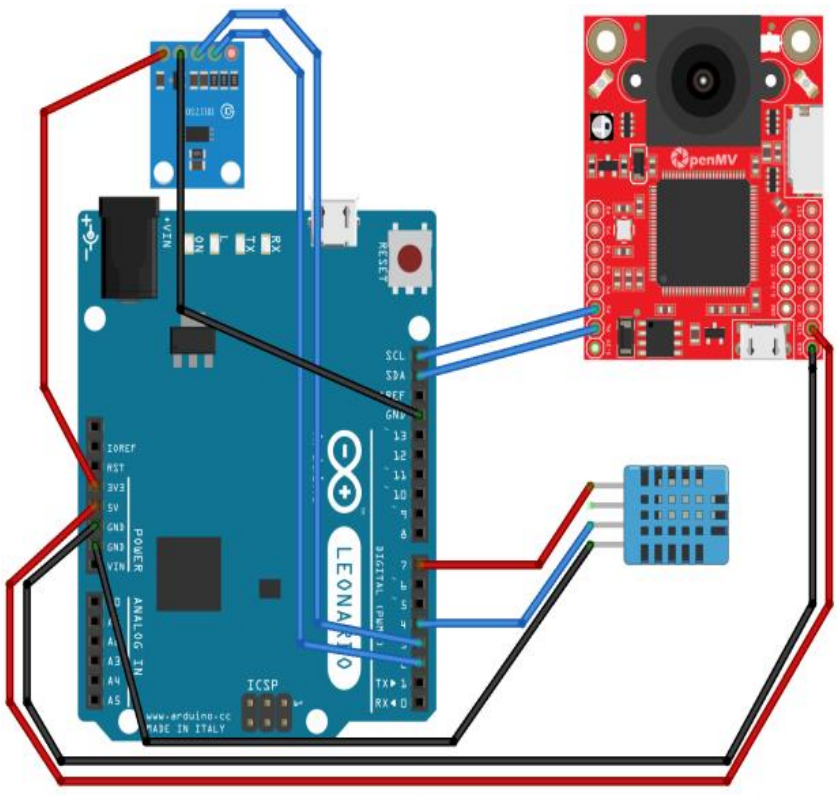

Figure (4) The circuit diagram of hardware

fritzing

\subsection{Arduino Leonardo board}

Arduino Leonardo board is responsible for controlling modules, receiving data and communicating with the thing network. The function carried out includes driving light density sensor, temperature and humidity sensor and OpenMV and upload this information to the thing network.

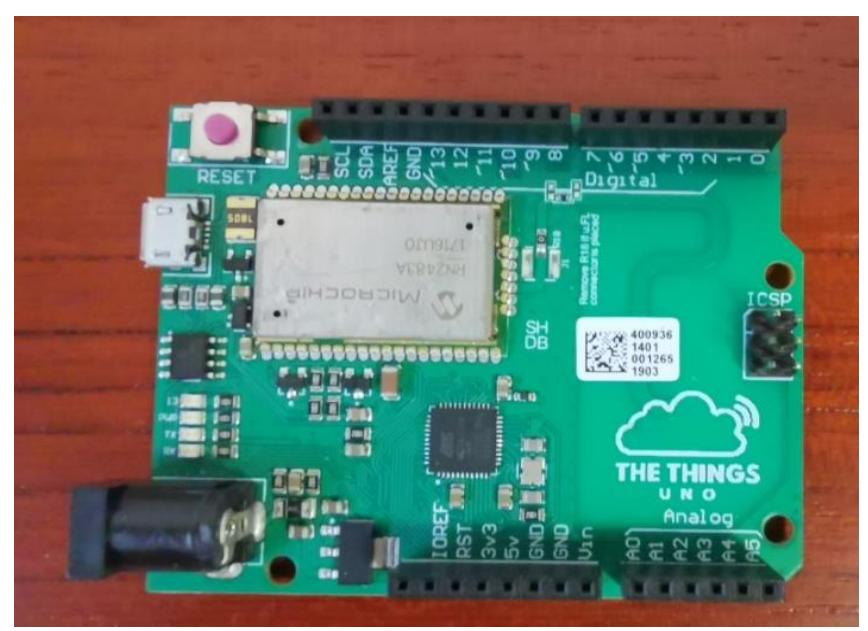

Figure (5) The Arduino Leonardo board

\subsection{Camera module}

OpenMV is a very significant part in this system. This module is compiled by micropython. When freezer door opened, GY-302 will sense changed in brightness and switch on the camera module. Through the built-in CNN algorithm of the OpenMV, category recognition and barcode recognition can be achieved.

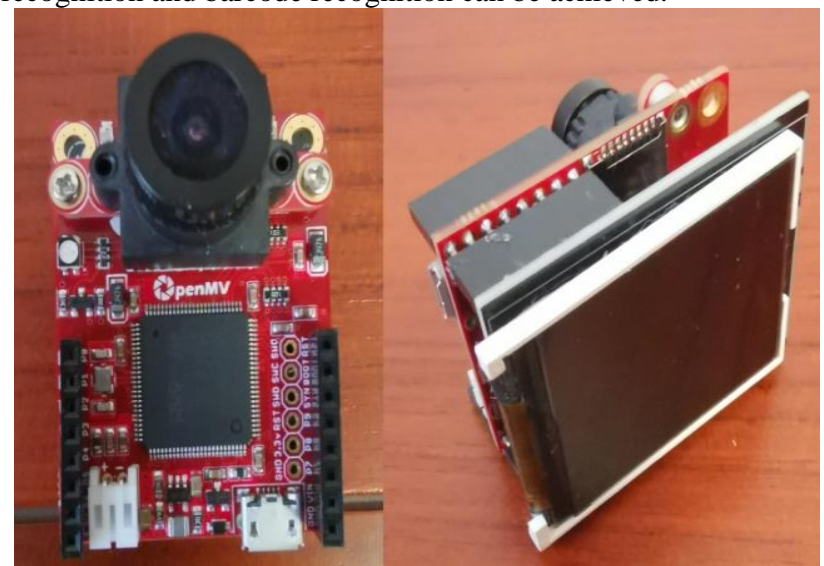

Figure (6) OpenMV camera module

\subsubsection{Food category recognition}

The development of the image recognition model for food types is based on the fruit-360 data set. This data set contains 8,000 photos of 120 fruits and vegetables. The picture format is $100 * 100$ pixels. Convert the downloaded binary file into a LMDB format file through the Caffe program. LMDB is a type of data label. Setting the label is helpful for Caffe to increase the speed when reading, and it is also convenient for the standardization of the training model. Then set the layer and related parameters of the convolutional neural network in Caffe. In this experiment, the model obtained after training 5000 times was used.

After deploying the model on OpenMV, the effectiveness of the model was verified by testing oranges, apples and bananas and achieving successful identification.

\subsubsection{Barcode recognition}

Since the color is very significantly affected by natural conditions such as light, the barcode image obtained by OpenMV must be processed by image binarization. The binarization of the image greatly reduces the amount of data in the image, which can highlight the outline of the target. Using this method to filter the graphics can effectively offset the negative effect of the lights in the refrigerator on the recognition. The barcode recognition program built in OpenMV can distinguish different types of barcodes and send the recognized barcode characters to the Arduino Leonardo board through serial communication.

\subsection{The Thing Network and Ubidots}

The Internet based sub-system achieves the interaction between system and people. The data collected by On-board sub-system will upload to Gateway by Arduino Leonardo board including the real time temperature, the category of food, the expiration date and the barcode. The information are imported into Ubidots. Here, this website would send message to the user if the event set in 
program is triggered consisting of excessive internal temperature and stored food exceeds expiration date. There is a list of the type of food which help users view through the interface easily. Barcode will also save in here which means the food supplier can obtain this information and support more personalized services to customers.

\section{:̊: Ubidots}

\section{E Ursalink_T7}

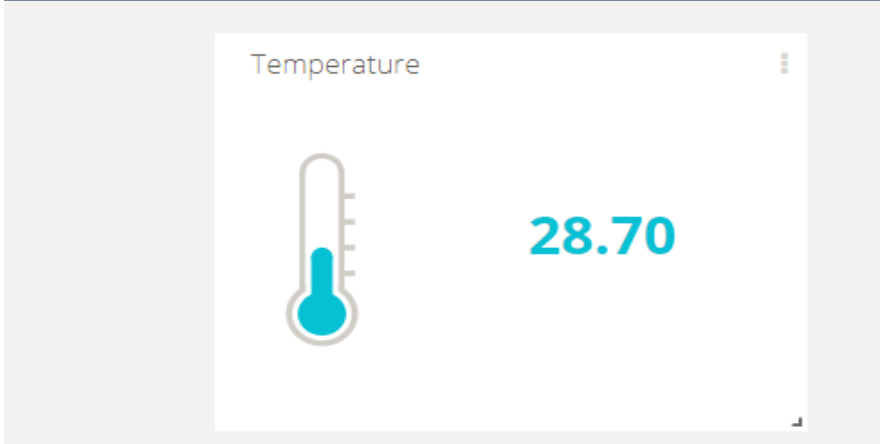

Figure (7) The Ubidots dashboard in room temperature

\subsection{DHT11 Temperature \& Humidity sensor}

DHT11 digital temperature and humidity sensor is a temperature and humidity composite sensor with calibrated digital signal output. An 8-bit single-chip microprocessor controls a resistive humidity sensing element and an NTC temperature measuring element. DHT11 uses a single bus protocol to communicate with the microcontroller. The data is 5 bytes consisting of 8 -bit humidity integer data +8 -bit humidity decimal data +8 -bit temperature integer data +8 -bit temperature decimal data +8 -bit checksum. Since the resolution of DHT11 can only be accurate to one digit, the fractional part is all zero. The checksum is the sum of the first 4 bytes of data. The purpose of the inspection is to ensure the accuracy of data transmission [7].

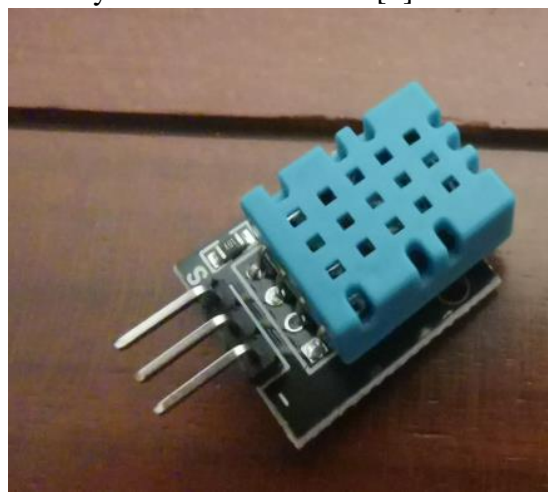

Figure (8) The DHT11 model

\subsection{LCD display}

Through the LCD screen connected to OpenMV, the user can observe whether the image is shot correctly.

\section{RESULT}

This system implements real-time monitoring of internal temperature of the refrigerator, identifies barcodes, expiration dates and category of fruits, uploads the information to the thing network and imports the information into Ubidots for sending message and displaying temperature on dashboard.

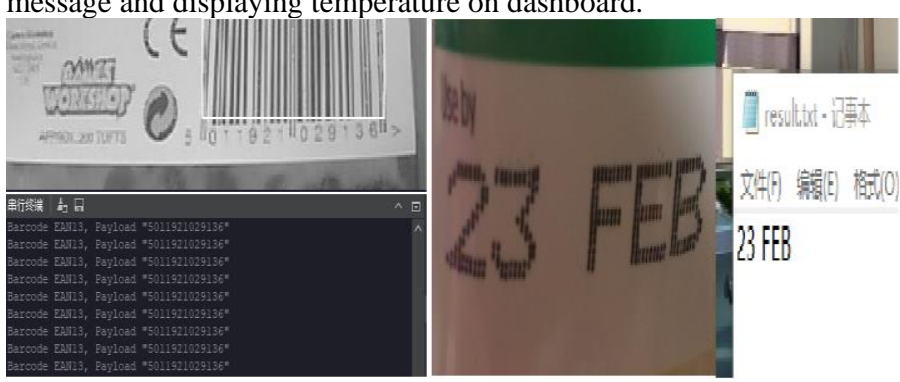

Figure (9) Result - Acquiring the barcode and date(s)

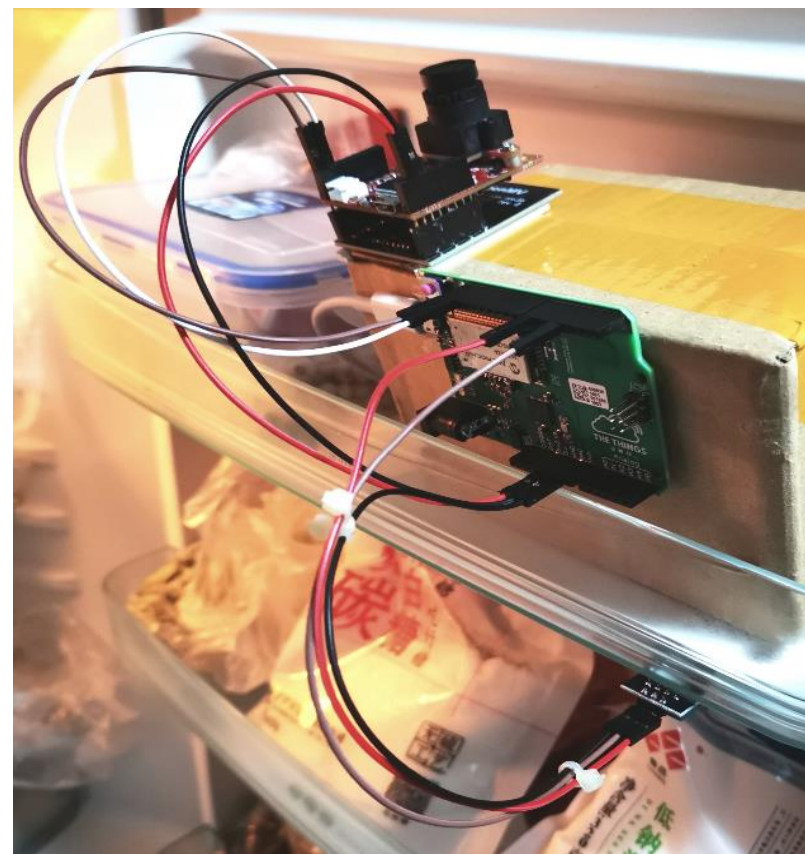

Figure (10) Working prototype

\section{CONCLUSION}

This intelligent refrigerator management system developed based on Artificial Intelligence technology is to enable users to manage household refrigerators more effectively with the support of the Internet of Things. This system composes On-board sub-system and Internet based sub-system. The machine vision OpenMV module obtains the barcode and expiration date printed on the surface of package and identification the category of fruits. DHT11 sensor collects the temperature. Through the things network, users can check the real-time internal state of the refrigerator and receive the message of events such as excessive temperature. Internet of Things technology will also facilitate food supplier, as they can monitor the status of goods and develop more value-added services using barcode related information. One 
of the significant concepts of this system is to connect refrigerator to the internet, based on which some additional interesting functions and features may be added to the future models. Family nutritionists can provide remote healthy diet guidance through the list of storage support by this system. Moreover, the system can provide online shopping guides and information through learning user references automatically.

\section{ACKNOWLEDGEMENT}

Authors would like to acknowledge the support provided by Glasgow College, UESTC.

Authors would also like to acknowledge Sultan Qaboos University (Government of the Sultanate of Oman) for supporting Dr. Amir M. Abdulghani.

\section{REFERENCES}

[1] Davenport M L, Qi D, Roe B E, et al. "Food-related routines, product characteristics, and household food waste in the United States: A refrigerator-based pilot study[J]." Resources Conservation and Recycling, 2019.

[2] C. Zhang, R. Green, "Communication security in Internet of Thing: Preventive measure and avoid DDoS attack over IoT network", Proc. 18th Symp. Commun. Netw. (CNS), pp. 8-15, 2015.

[3] T. Brown and N.A. Hipps, "Reducing domestic food waste by lowering home refrigerator temperatures." [Online], Available: https://www.sciencedirect.com/science/article/abs/pii/S0140 700713003629

[4] Romero, Alejandro, and Elisabeth Lefebvre. "Combining barcodes and RFID in a hybrid solution to improve hospital pharmacy logistics processes." International Journal of Information Technology and Management 14.2 (2015): 97123.

[5] Luo Xiaonan, Zhang Zongwei, Lin Mouguang, "Food management and recommendation system based on intelligent refrigerator" Applied Energy (2013)

[6] Zheng Shuangming, Wang Mingguo, "Refrigerator food management method and system, server and refrigerator" Applied Energy (2013)

[7] Fei, Wang. "Research application of the digital temperature and humidity sensor DHT11." Electronic Design Engineering (2013).

[8] Li Ce, "Research and Implementation of Food Recognition Mobile System Based on Deep Visual Perception Learning. " Computer and Telecom, (2019)

[9] Cengil, E., Çınar, A., \& Özbay, E. (2017). "Image classification with caffe deep learning framework." In 2017
International conference on computer science and engineering (UBMK), Antalya (pp. 440-444).

[10] Chen Haifeng and Ding lili, "Research on Gray Processing Algorithm of Binary Image [J]." Computer and Telecom, 2019 (07): 34-38. 